\title{
Large velocity fluctuations in small-Reynolds-number pipe flow of polymer solutions
}

\author{
D. Bonn, ${ }^{1,2}$ F. Ingremeau, ${ }^{3}$ Y. Amarouchene, ${ }^{3}$ and H. Kellay ${ }^{3}$ \\ ${ }^{1}$ Laboratoire de Physique Statistique de l'ENS, 24 rue Lhomond, F-75005 Paris, France \\ ${ }^{2}$ Van Der Waals-Zeeman Institute, Université of Amsterdam, Valckenierstraat 65, NL-1018XE Amsterdam, The Netherlands \\ ${ }^{3}$ Laboratoire Ondes et Matière d'Aquitaine, U. Bordeaux 1, UMR 5798 CNRS, 351 cours de la Libération, F-33405 Talence, France
}

(Received 23 February 2011; revised manuscript received 13 September 2011; published 10 October 2011)

\begin{abstract}
The flow of polymer solutions is examined in a flow geometry where a jet is used to inject the viscoelastic solution into a cylindrical tube. We show that this geometry allows for the generation of a "turbulentlike" flow at very low Reynolds numbers with a fluctuation level which can be as high as $30 \%$. The fluctuations increase with an increase in solution polymer concentration and flow velocity. The turbulent fluctuations decay downstream for small flow velocities but persist for high velocities. The statistical properties of the generated fluctuations indicate that this turbulentlike flow is different from previously studied flows displaying elastic turbulence and shows a direct cascade of energy to small scales with practically no intermittency.
\end{abstract}

DOI: 10.1103/PhysRevE.84.045301

PACS number(s): 47.20.Gv, 47.27.nf, 47.50.-d

In Newtonian fluids, turbulence sets in at high enough Reynolds numbers, a measure of the ratio of inertial to viscous forces. It is given by $\operatorname{Re}=U d / \nu$, where $v$ is the kinematic viscosity, $U$ a large-scale velocity, and $d$ an appropriate scale in the system [1]. For non-Newtonian fluids, the situation is less clear. The addition of small amounts of polymers to high-Reynolds-number flows can diminish the turbulent intensity dramatically, a phenomenon called turbulent drag reduction $[2,3]$. However, in the opposite limit of very small Reynolds numbers, flow instabilities [4] and even a random and chaotic motion of the fluid known as elastic turbulence [5] may also be observed for very similar dilute solutions of polymers. Such flow instabilities must find their origin in the non-Newtonian flow properties of the fluid, notably elastic forces that result from the stretching of the polymers by the flow $[4,5]$. The relevant dimensionless number is the Weissenberg number $\mathrm{Wi}=\dot{\gamma} \lambda$, which compares the velocity gradient $\dot{\gamma}$ in the fluid to the viscoelastic relaxation time of the polymer solution $\lambda$. Such low-Reynolds-number random flows may have applications for mixing chemical reactants in microchannels $[5,6]$ but may also be an unwanted side effect in the manufacturing of polymer fibers [7-9].

It is known that polymer solutions can become unstable in flows with curved streamlines provided the velocity is large enough [10]. The instability threshold depends on the curvature of the streamlines [10]. This naturally poses the question of what happens for the more usual case of flows in straight channels, without any curvature, such as in microchannels. A linear stability analysis shows that these flows are linearly stable [11]; however, a recent nonlinear stability analysis shows that a finite-amplitude perturbation of the flow may lead to instability for large enough Wi $[7,8,12]$ : The instability is subcritical.

In this Rapid Communication we present evidence for the existence of "elastic turbulence" in straight channels, and also show that a prerequisite for observing such unstable flows is a very large initial perturbation of the flow. This concurs with the theory, and provides a plausible reason why, to the best of our knowledge, the effect has not been observed before, in spite of a large number of efforts. In addition, we characterize the flow and show that it has properties usually associated with large-Reynolds-number flows, such as the presence of an energy cascade and the scaling of the structure functions.

The experiments use a large molecular weight polymer $\left(18 \times 10^{6}\right.$ amu polyacrylamide $)$ in water at $10-\mathrm{mM} \mathrm{NaCl}$ at concentrations between 100 and $1000 \mathrm{ppm}$. The rheology of the solutions was done with a cone-plate geometry in a commercial rheometer (Rheologica). This allows to determine the relaxation time $\lambda$ and shear-rate-dependent viscosity of the solutions [Fig. 1(a)]. For the flow experiments, solutions are injected in cylindrical tubes of different cylinder diameters $(2 \mathrm{R}=5$ and $8 \mathrm{~mm})$ using a syringe pump, after its passage through a smaller tube ( 1 or $3.5 \mathrm{~mm}$ diam) to produce a perturbation of the flow where the small tube enters the larger one. The velocity and its fluctuations are measured using a laser Doppler velocimeter (LDV) (probing a zone of $0.1 \mathrm{~mm}$ in diameter and $0.8 \mathrm{~mm}$ in length) at or near the centerline of the tubes. We measure the velocity of tracer particles ( 1 or 6 $\mu \mathrm{m}$ in diameter) with which the solution is seeded, allowing for a measurement of the time series of the longitudinal velocity at different distances $x$ from the exit of the small tube. Control experiments were carried out using water-glycerol solutions of shear viscosity $400 \mathrm{mPa} \mathrm{s}$, comparable to the zero shear rate viscosity of the most concentrated polymer solutions. The velocity time series is then analyzed to extract the level of fluctuations, the velocity histograms, and the power spectra of the temporal velocity variations.

The first remarkable feature is the much larger level of velocity fluctuations for the polymer solution as compared to the control solution as shown in Fig. 1(b). The two solutions have comparable shear viscosities corresponding to Reynolds numbers of 0.08 and 0.04 , respectively. However, the polymer solution exhibits large velocity fluctuations with an intensity (width of the histogram at half maximum divided by the mean value) of nearly $30 \%$ as compared to only $3 \%$ for the control case. The presence of large fluctuations is further illustrated by the power spectra of velocity fluctuations shown in Fig. 1(c). Here, the power spectrum of the glycerol solution is flat and comparable to the noise floor of the measurements. For the polymer case, at comparable injection flux, the power spectra are broad band and present at least a two order of magnitude increase with respect to the test solution at the low-frequency 

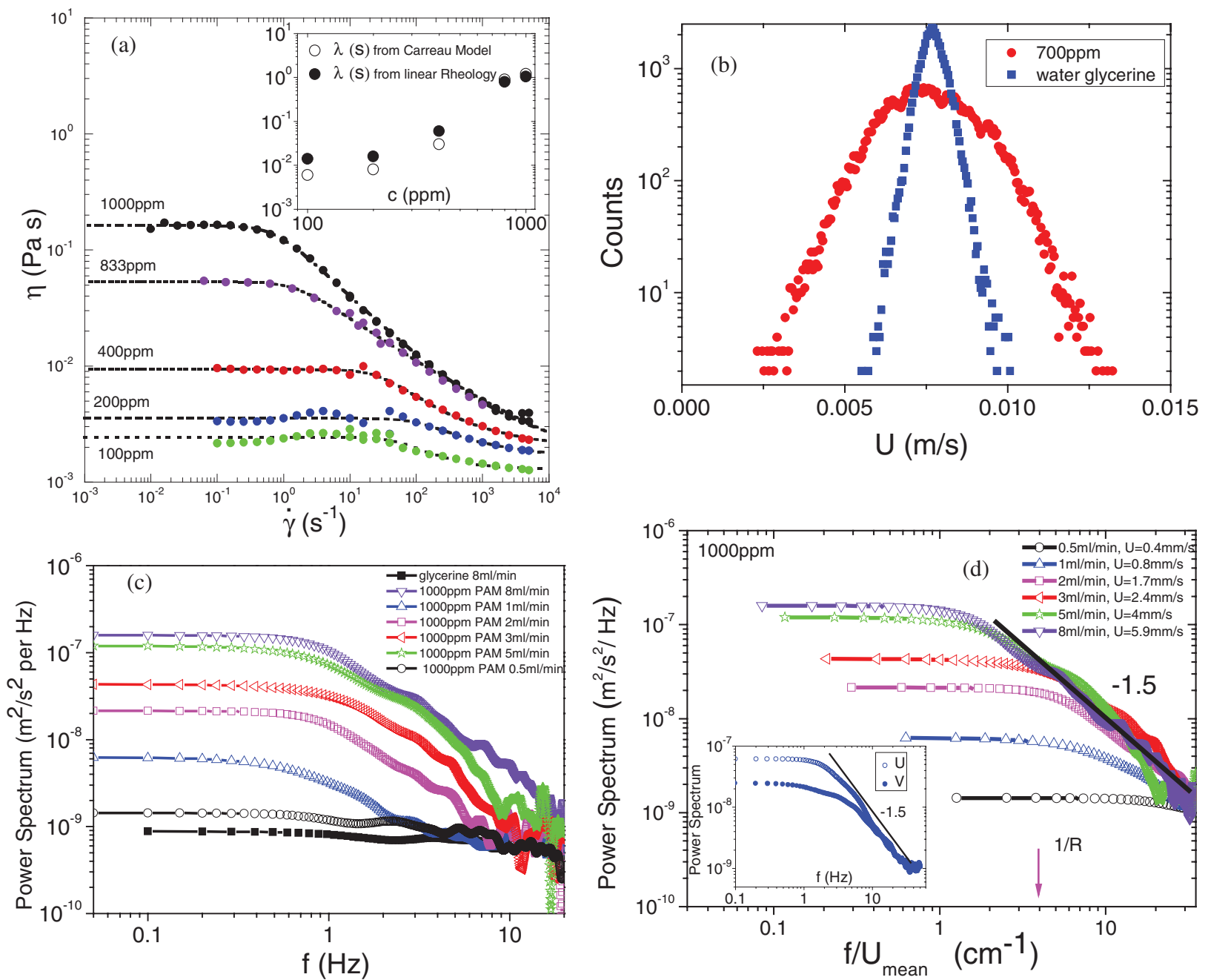

FIG. 1. (Color online) (a) Viscosity vs shear rate. The relaxation times $\lambda$ were obtained using a Carreau model (dashed lines) and linear rheology measurements. (b) Histograms of the velocity at $x=3.5 \mathrm{~cm}$ for a flux of $8 \mathrm{ml} / \mathrm{min}$. (c) Power spectra at $x=3.5 \mathrm{~cm}$. (d) Spectra vs wave number the arrow indicates the radius of the channel. Inset: Spectra of the longitudinal and transverse components.

end of the spectrum. By reducing the injection flux and therefore the mean velocity of the flow, the fluctuation level decreases and the spectral amplitude decreases accordingly. However, the spectra always remain at significantly higher fluctuation levels than the control solution. These observations are also recovered when the polymer concentration decreases; here the spectral amplitude decreases with concentration. The large fluctuations we observe therefore increase when the polymer concentration and the mean flow velocity increase.

A further analysis of the spectra is shown in Fig. 1(d), where the frequency axis is normalized by the mean velocity of the flow. In high-Reynolds-number turbulence this procedure amounts to admitting the validity of the so-called Taylor frozen turbulence hypothesis. This hypothesis has not been tested explicitly here, but for viscoelastic flows in the elastic turbulence regime it was found to work reasonably well when the mean flow is well defined [13]. Figure 1(d) suggests that the high-frequency part of the spectra for different injection fluxes but similar concentration seems to asymptote to the same level. This asymptote can be roughly approximated by a power-law decay with an exponent 1.5 for scales smaller than the radius of the channel R. The inset of Fig. 1(d) shows the power-law decay of the spectrum for both the longitudinal and transverse component of the velocity, indicating anisotropy of the large scales but isotropy at the small-scale (high-frequency) end of the spectrum.

A second observation concerns the evolution of the spectra as the measurement position varies. We measure the fluctuations at different distances from the entrance tube (the flow perturbation) and observe that for small Wi (typically Wi $=1$ ) the fluctuation intensity decreases with the downstream distance from the entrance $x$ when Wi is small; however, a high level of fluctuations persists when Wi is high. This is illustrated in Fig. 2(a) for two values of $\mathrm{Wi}, 0.7$ and 4. The distance $y$ divided by the mean flow velocity gives us the time evolution (persistence) of the turbulence. For low Wi, it decays away from the perturbation, which is reminiscent of decaying jet or grid turbulence; however, the decay is much slower than a simple flow perturbation in a laminar flow and seems to be algebraic. Note that this decay occurs over a period of time that is at least ten times longer than the polymer relaxation time $\lambda \approx 1 \mathrm{~s}$ before the fluctuations reach the noise floor. The decay 

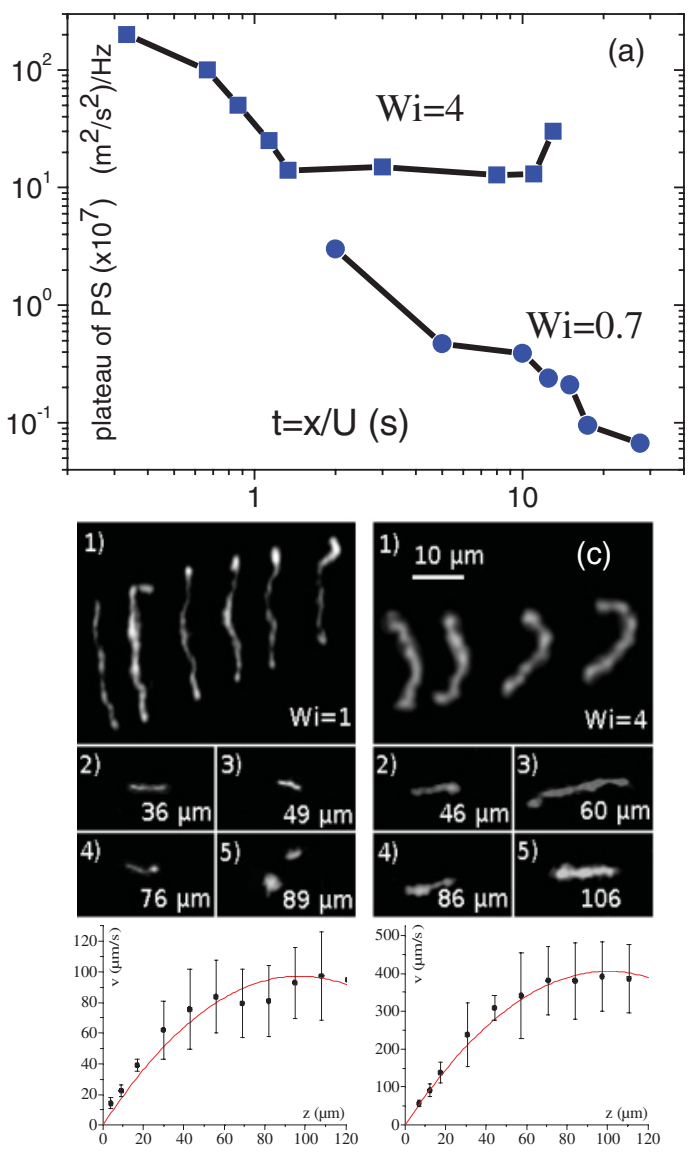
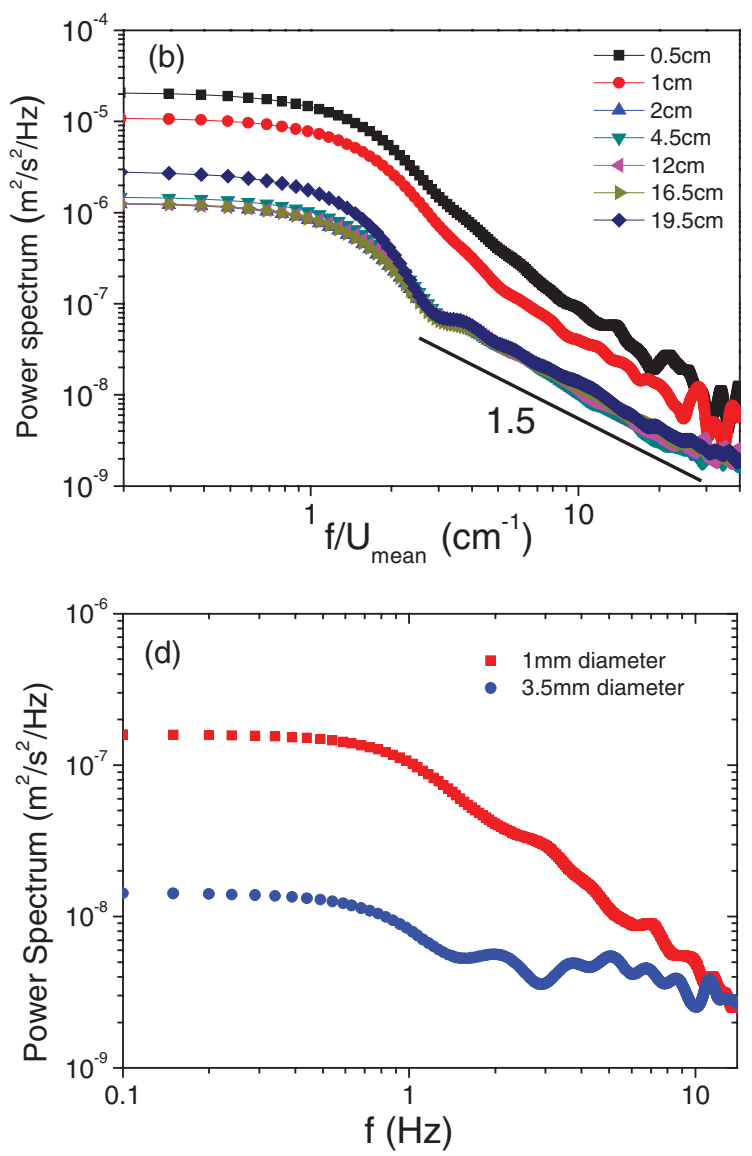

FIG. 2. (Color online) (a) Amplitude of fluctuations vs $t=x / U$ for a 1000-ppm solution. (b) Power spectra at different $x$ for Wi $=4$. (c) Polymer elongations for two Wi: Image 1 is near the outlet, and images 2-5 are farther down stream and at different distance $z$ (indicated in the images) from the wall of the channel. Velocity profiles and a parabolic fit: The vertical bars indicate the fluctuation level. (d) Spectra using two different injection diameters.

of the fluctuations for small Wi suggests that the fluctuations introduced by the small tube die out over time and that the flow will become stable farther downstream. The persistence of the fluctuations for large $\mathrm{Wi}$, on the other hand, suggest a sustained state. Very close to the exit of the small tube the fluctuations decay somewhat, but farther downstream the decay is arrested signaling that the fluctuations persist: A plateau value of the amplitude is obtained [Fig. 2(a)]. Figure 2(b) shows the spectra, for this value of $\mathrm{Wi}$, taken at different distances from the exit of the small tube. The spectra show a fast decrease in amplitude at first, but farther away, they remain practically unaltered when changing the downstream distance. The scaling of the high-frequency end also persists.

To gain more insight into the microscopic dynamics of the polymers, we carried out additional experiments to examine the polymer conformations within the flow. In the pioneering work of Steinberg and collaborators, polymers exhibited strong elongations in the elastic turbulence regime. These elongations can be used as a measure of the local elastic stresses in the flow [14]. To carry out a visualization of the polymer conformations, we seeded a 1000-ppm polymer solution prepared in an appropriate buffer with a small quantity of fluorescent T4 DNA molecules [15]. To be able to do experiments under a microscope, the flow glass cell now consists of a 2-cm-long rectangular channel of $200 \mu \mathrm{m}$ height and $2 \mathrm{~mm}$ width into which a cylindrical tube of $100 \mu \mathrm{m}$ diam was inserted for the injection of the solution. This cell was mounted on an inverted microscope equipped with an oil immersion objective [63× numerical aperture (NA) 1.46] and a sensitive camera [EMCCD], allowing to directly visualize the conformation of the DNA polymers. We observe strong elongations within the small cylindrical injection tube. At the outlet of this tube, the streamlines of the flow show a strong divergence, and the polymers exiting the tube are observed to be either already elongated or to get stretched by the diverging streamlines as shown in the two upper images of Fig. 2(c) for two different values of Wi. We estimate that the polymers can be elongated up to at least $50 \%$ of their total length; in doing so, they exhibit a variety of conformations, notably elongation both parallel and perpendicular to the main flow direction. We subsequently examine the elongation of the polymers at different heights $z$ in the rectangular channel and at different positions in the flow direction for two different $\mathrm{Wi}, 1$ and 4. The small Wi flow shows that the polymers may be elongated near the outlet of the cylindrical tube and near the walls of the channel farther downstream (image 2) but not near the middle of the channel (image 5) where the shear rate is small: See the corresponding velocity profiles in Fig. 2(c) (the velocity fluctuations around the mean in these measurements obtained from particle tracking are near $30 \%$, which is consistent with 


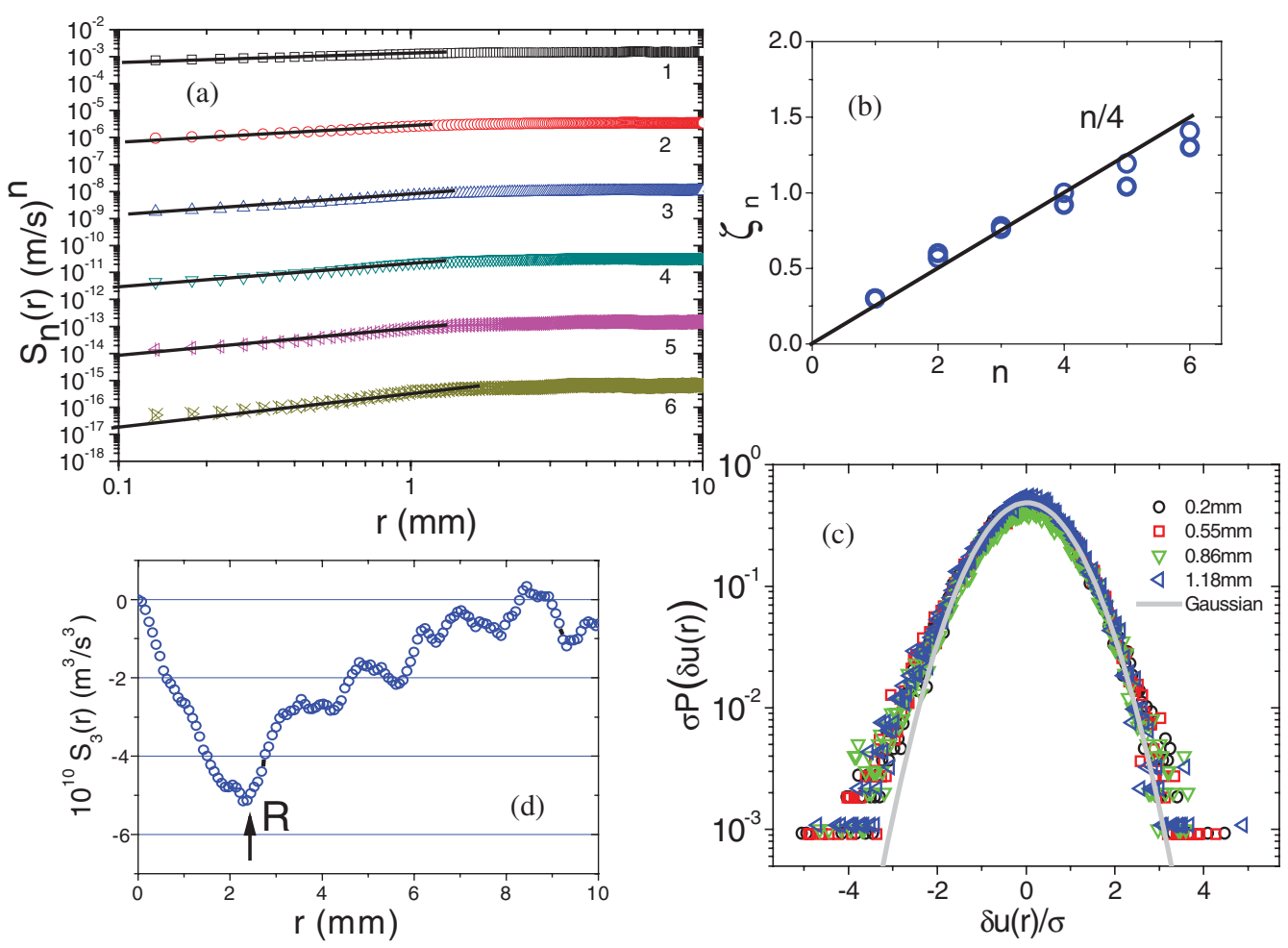

FIG. 3. (Color online) (a) $S_{n}(r)$ for $1000 \mathrm{ppm}$ at $8 \mathrm{ml} / \mathrm{min}$. (b) $\zeta_{n}$ obtained from different runs are reported. (c) The pdf's of velocity increments and a Gaussian fit. (d) $\mathbf{S}_{3}(r)$.

the laser Doppler velocimeter (LDV) measurements). The snapshots in Fig. 2(c) are taken at different heights in the channel for a distance of $1 \mathrm{~cm}$ from the outlet, i.e., sufficiently far from the outlet so that entrance effects can be neglected. Note that near the center of the channel, the molecules are not strongly elongated. For the higher Wi flow, strong elongations, even in the middle of the channel where average shear rate is small, are clearly observed (images 4 and 5 for $\mathrm{Wi}=4$ ). The elongations of the polymers observed here persist as the distance to the entrance increases for high Wi but not for the low Wi case. We conclude that both the passage in the small tube and the diverging flow at its exit contribute to elongate the polymers and therefore inject elastic stresses into the flow. These elastic stresses then drive the turbulent flow; the latter persist farther downstream for high $\mathrm{Wi}$, and die out for small Wi, in agreement with the macroscopic measurements presented above.

These observations suggest that for high enough $\mathrm{Wi}$, large fluctuations persist. However, this is not the only condition: The injection also has to be violent enough. This follows from our observation that different injection schemes lead to very different results. We use different injection tubes of 1 and $3.5 \mathrm{~mm}$ diam. The power spectrum in the channel in the first case has an amplitude at least an order of magnitude larger than in the second case, signaling that the injection through a smaller tube is much more efficient in producing the large fluctuations [Fig. 2(d)] and that the flow is more stable if the perturbation at the entrance is smaller. This ties in with recent theoretical analyses of viscoelastic channel flow [7,12], where the subcritical nature of the instability leads to the prediction that the flow goes unstable only if the initial perturbation is strong enough and for Wi greater than a threshold value of $\sim 5$. Our experiments show that first, a strong perturbation is needed to render the flow "turbulent" and that this "turbulence" decays downstream for small Wi while it persists for higher Wi.

Let us now focus on the properties of this turbulence. The statistics of the fluctuations are usually characterized by the structure functions of the absolute value of velocity differences across different time scales or length scales $r$ if we again invoke the Taylor hypothesis $S_{n}(r)=\left\langle|U(x+r)-U(x)|^{n}\right\rangle$. This analysis confirms the scaling behavior of the power spectrum at high frequencies observed for the high concentration solutions. This follows from the power-law scaling of the structure functions with $r$ as well as from the variation of the exponents $\zeta_{n}$ versus the order $n$ of the structure functions, which for an exponent of 1.5 for the spectrum should vary as $n / 4$, shown in Figs. 3(a) and 3(b). A remarkable feature here is that the exponents seem to vary roughly linearly with $n$ with only a mild deviation for the fifth and sixth moments and no noticeable changes with respect to Wi. Despite this mild deviation, the probability density functions (pdf's) of velocity differences remain roughly the same all through the scaling range, as shown in Fig. 3(c) where they are found to superimpose for different values of $r$ (indicated by solid lines in Fig. 3(a)) showing that the functional shape of these pdf's is independent of the increment $r$. Interestingly, these functions do show a skewness toward negative velocity differences. An attempt at fitting these pdf's with a Gaussian function show that while this function may approximate the central portion of the pdf's, it fails for the negative velocity tail due to the asymmetry. A final remark concerns the third-order moment of velocity increments $\mathbf{S}_{3}(r)=\left\langle[U(x+r)-U(x)]^{3}\right\rangle$. This moment is 
negative (in agreement with the observation of the skewed pdf) and increases in absolute value as the scale increases, before going through a minimum at a scale comparable to the radius of the pipe used [Fig. 3(d)]. These features indicate that, despite the obvious differences between the two flows, this low-Reynolds-number flow exhibits features in common with high-Reynolds-number turbulent flows such as a negative third moment and therefore a nonzero energy transfer rate from large to small scales.

For elastic turbulence in curved channels, previous experiments, supported by theory [16] and numerical simulations [17], suggest a power-law scaling of the power spectra as well; however, the important difference is that the exponent found is near $3.3[5,18]$. Experiments in a Taylor-Couette cell showed exponents smaller than 3 [18], suggesting that the type of base flow may also modify the statistical properties of the velocity fluctuations. Here, we find an exponent that is significantly smaller, which suggests a different "type" of turbulence in our linear channel. In fact, an exponent smaller than 3 suggests that the velocity field is singular over the scaling range [19]. Our observation of power-law scaling with a power smaller than 3 is valid for the three concentrations we have examined. However, the exponent seems to increase from 1.5 at $1000 \mathrm{ppm}$ to nearly 3 at $200 \mathrm{ppm}$ : If the polymer concentration decreases, the velocity field seems to become smooth. In addition to the linearity of our channel, it is then probable that the difference between our findings and previous results [5] is due to the high concentrations used here and perhaps also to the shear thinning character of our solutions.

We conclude from the above observations that lowReynolds-number pipe flow of polymer solutions may become unstable and turbulentlike if the flow is perturbed sufficiently. The results indicate that the turbulentlike flow has different properties from previously found random flows in viscoelastic solutions. It may well be that the geometry of the flow is at the heart of such differences. We speculate that such fluctuations of a different nature than seen previously will also change the way mixing is achieved in low-Reynolds-number flows of viscoelastic solutions.

We would like to thank V. Steinberg for a critical reading of the manuscript.
[1] W. D. McComb, The Physics of Fluid Turbulence (Oxford University Press, Oxford, UK, 1990).

[2] A. Gyr and H. W. Bewerdsdorff, Drag Reduction of Turbulent Flows by Additives (Kluwer, Amsterdam, 1995).

[3] D. Bonn et al., J. Phys. Condens. Matter 17, 1195 (2005).

[4] S. J. Muller, R. G. Larson, and E. S. G. Shaqfeh, Rheol. Acta 28, 499 (1989).

[5] A. Groisman and V. Steinberg, Nature (London) 410, 905 (2001); Phys. Rev. Lett. 86, 934 (2001).

[6] T. Burghelea, E. Segre, I. Bar-Joseph, A. Groisman, and V. Steinberg, Phys. Rev. E 69, 066305 (2004).

[7] B. Meulenbroek et al., Phys. Rev. Lett. 90, 024502 (2003).

[8] V. Bertola et al., Phys. Rev. Lett. 90, 114502 (2003).

[9] M. Denn, Annu. Rev. Fluid Mech. 22, 13 (2000).

[10] P. Pakdel and G. H. McKinley, Phys. Rev. Lett. 77, 2459 (1996).
[11] C. Ho and M. M. Denn, J. Non-Newtonian Fluid Mech. 3, 179 (1978).

[12] A. N. Morozov and W. van Saarloos, Phys. Rev. Lett. 95, 024501 (2005).

[13] T. Burghelea, E. Segre, and V. Steinberg, Phys. Fluids 17, 103101 (2005)

[14] Y. Liu and V. Steinberg, Europhys. Lett. 90, 44002 (2010).

[15] N. François, D. Lasne, Y. Amarouchene, B. Lounis, and H. Kellay, Phys. Rev. Lett. 100, 018302 (2008); Europhys. Lett. 86, 34002 (2009).

[16] A. Fouxon and V. Lebedev, Phys. Fluids 15, 2060 (2003).

[17] S. Berti, A. Bistagnino, G. Boffetta, A. Celani, and S. Musacchio, Phys. Rev. E 77, 055306(R) (2008).

[18] A. Groisman and V. Steinberg, New J. Phys. 6, 29 (2004).

[19] U. Frisch, Turbulence, The Legacy of A. N. Kolmogorov (Cambridge University Press, Cambridge, UK, 1995). 